\title{
Deuteron electromagnetic form factors in the Light-Front Dynamics
}

\author{
J. Carbonell ${ }^{*}$ \\ Institut des Sciences Nucléaires, 53 avenue des Martyrs, 38026 Grenoble Cedex, France \\ V.A. Karmanov ${ }^{\dagger}$ \\ Lebedev Physical Institute, Leninsky Prospekt 53, 117924 Moscow, Russia
}

October 16, 2018

\begin{abstract}
The deuteron form factors are calculated in the framework of the relativistic nucleonmeson dynamics, by means of the explicitly covariant light-front approach. The inflluence of the nucleon electromagnetic form factors is discussed. At $Q^{2} \leq 3(\mathrm{GeV} / \mathrm{c})^{2}$ the prediction for the structure function $A\left(Q^{2}\right)$ and for the tensor polarization observable $t_{20}$ are in agreement with the recent data of CEBAF/TJNAF.
\end{abstract}

\section{Introduction}

Recently the first experimental data obtained at Thomas Jefferson National Accelerator Facility (former CEBAF) on the deuteron structure function $A$ and tensor polarization $t_{20}$, measured in the elastic ed scattering, have been reported [1 14 . They are rather precise and correspond to a maximum momentum transfer of $6(\mathrm{GeV} / \mathrm{c})^{2}$. Since in the ed collision the deuteron gets from the electron a momentum comparable with the deuteron mass, these data are probing the trully relativistic dynamics inside the deuteron.

Our preliminary calculations of the form factors and of $t_{20}$ have been presented in [5]. We present in this paper the details of our approach, discuss the influence of the different choices of nucleon electromagnetic form factors parametrization and compare our results with the reported experimental data.

In our calculations we assume the deuteron structure to be determined by the relativistic nucleon-meson dynamics. Namely, we suppose that the nucleons in the deuteron interact by exchanging relativistic mesons. We take the same set of mesons and parameter values used in the construction of the Bonn potential [6]. However, we do not make any nonrelativistic potential approximation and calculate the one-boson-exchange kernel in relativistic form, as it appears from field theory.

In the momentum tranfer region scanned in TJNAF, the relativistic effects related to the nucleons motion, to the spin rotations and to the retardation of the exchanged mesons,

*e-mail: carbonel@isn.in2p3.fr

${ }^{\dagger}$ e-mail: karmanov@sci.lebedev.ru 
should manifest themselves in full measure and be of crucial importance in describing the data. Therefore we believe that their main part can be properly taken into account even in an approximate relativistic calculation. On the other hand, it is important to work in an approach which provides a clear physical interpretation of the incorporated effects.

The deuteron form factors are calculated in the framework of the explicitly covariant version of the Light-Front Dynamics (LFD) recently reviewed in [5]. In this approach, the state vector is defined on the light-front plane of general position $\omega \cdot x=0$, where $\omega$ is a four-vector such as $\omega^{2}=0$. This restores the relativistic covariance lost in the standard light-front approach which is obtained as a particular case for $\omega=(1,0,0,-1)$. The relativistic wave functions the Fock components of the state vector - are the closest couterparts of the nonrelativistic ones. This allows one to benefit from the knowledge of the nonrelativistic wave functions and to incorporate selfconsistently the relativistic effects. Using the light-front plane leads to significant simplifications due to the absence of vacuum fluctuations. The diagram technique is a three-dimensional one and can be interpreted in terms of time-ordered physical processes. Therefore the selection of the diagrams contributing to the kernel or to the form factors, whose numerical estimation is rather difficult in any approach, can be supported by the intuitive understanding of the corresponding physical process. All that allows, in a given relativistic dynamics, to carry out the calculations on a satisfactory level of confidence.

We mention also few other approaches to calculate the relativistic wave function and the deuteron electromagnetic form factors. In the standard version of LFD, defined on the plane $t+z=0$, these form factors were calculated in [7- 10]. The results obtained in [11, 12] are based on the Bethe-Salpeter approach and the works [13, 14 use a three-dimensional reduction of the Bethe-Salpeter function - the Gross wave function [15].

In sect. 2 we present the relativistic deuteron wave function on the light front. In sect. 3 the electromagnetic vertex of the deuteron in the impulse approximation, based on the one-body electromagnetic nucleon current, is discussed. In sect. Tt we discuss the contact (instantaneous) interaction, corresponding to the $N N B \gamma$ vertex ( $B$ is an exchanged meson) which is a correction to the one-body current. In sect. 5 we explain how to extract the form factors, separating them from the nonphysical contributions. Section 6 is devoted to the evaluation of the nucleon electromagnetic form factors influence in the deuteron observables. Section 7 contains a comparison of our results with the experimental data and concluding remarks.

\section{Wave function}

The wave functions are the Fock components of the state vector defined on the light-front plane $\omega \cdot x=0$. The explicit covariance allows one to construct the general form of the light-front wave function for a system with a given spin. The relativistic deuteron wave function on the light front contains six spin components, in contrast to two components - S and D-waves in the nonrelativistic case. Its general form is given in [16, 17]. Below we will keep only three dominating components:

$$
\begin{aligned}
\Psi_{\lambda}^{\sigma_{2} \sigma_{1}} & =\sqrt{m} e_{\lambda}^{\nu}(p) \bar{u}^{\sigma_{2}}\left(k_{2}\right) \phi_{\nu} U_{c} \bar{u}^{\sigma_{1}}\left(k_{1}\right), \\
\phi_{\nu} & =\varphi_{1} \frac{\left(k_{1}-k_{2}\right)_{\nu}}{2 m^{2}}+\varphi_{2} \frac{1}{m} \gamma_{\nu}-\varphi_{5} \frac{i}{m^{2} \omega \cdot p} \gamma_{5} \epsilon_{\nu \alpha \beta \gamma} k_{1}^{\alpha} k_{2}^{\beta} \omega^{\gamma},
\end{aligned}
$$


where $p$ and $k_{1,2}$ are the on mass shell deuteron and the nucleon momenta, $e_{\nu}^{\lambda}(p)$ is the deuteron polarization vector, $\bar{u}^{\sigma}(k)$ is the nucleon spinor, $U_{c}$ is the charge conjugation matrix. We notice that the wave function defined on the light-front plane depends on the orientation of this plane through the argument $\omega$.

In the system of reference where $\vec{k}_{1}+\vec{k}_{2}=0$ the function (11) obtains the more transparent form:

$$
\begin{aligned}
\Psi_{\sigma_{2} \sigma_{1}}^{\lambda}(\vec{k}, \vec{n}) & =\sqrt{m} w_{\sigma_{2}}^{\dagger} \psi^{\lambda}(\vec{k}, \vec{n}) \sigma_{y} w_{\sigma_{1}}^{\dagger}, \\
\vec{\psi}(\vec{k}, \vec{n}) & =f_{1} \frac{1}{\sqrt{2}} \vec{\sigma}+f_{2} \frac{1}{2}\left(\frac{3 \vec{k}(\vec{k} \cdot \vec{\sigma})}{\vec{k}^{2}}-\vec{\sigma}\right)+f_{5} \sqrt{\frac{3}{2}} \frac{i}{k}[\vec{k} \times \vec{n}],
\end{aligned}
$$

where $\vec{k}$ is the value of $\vec{k}_{1}$ in this system of reference, $\vec{n}$ is the direction of $\vec{\omega}$ in this system, $w_{\sigma}$ is the two-component nucleon spinor. The scalar functions $f_{i}$ depend on the scalars $k \equiv|\vec{k}|$ and $z=\vec{n} \cdot \vec{k} / k$. One can find a special representation [17] in which the wave function (11) obtains the form (2) in arbitrary system of reference. The components $\varphi_{i}$ in (1) and $f_{i}$ in (2) are linearly related with each other [5, 17].

The wave function (11) was calculated in [17] in a perturbative way incorporating the full relativistic one-boson exchange (OBE) kernel found in LFD, with the corresponding nonrelativistic wave function as zero order approximation. This calculation can be considered as the perturbation theory developed in terms of the difference between the relativistic kernel and the nonrelativistic potential. For the OBE kernel the set of mesons, coupling constants and form factors corresponding to the Bonn model [6] were used. The solution thus obtained is approximate in two ways: first for its perturbative character does not provide an exact solution of the LFD equation and second because the parameters of the OBE kernel were kept to their original values i.e. were not fitted in the LFD relativistic framework. The accuracy of our solution in reproducing the low energy deuteron observables was estimated at the level of $20 \%$ in norm.

In nonrelativistic region of $k$, components $f_{1}$ and $f_{2}$ found this way turn into the usual S- and D-waves, whereas other components become negligible. However, starting from $k \approx 0.5$ $\mathrm{GeV} / \mathrm{c}$, component $f_{5}$ dominates over all other components, including $f_{1}$ and $f_{2}$.

The physical meaning of this dominating extra component has been clarified [18 by comparing, in $1 / m$ approximation, the analytical expression for the amplitude of the deuteron electrodisintegration near threshold with the nonrelativistic one, including meson exchange currents. For the isovector transition, in the region where the so called pair term with the pion exchange dominates, this component (together with a similar component in the scattering state) automatically incorporates $50 \%$ of the pair term contribution and therefore dominates too. Another $50 \%$ is given by the contact interaction (see below).

\section{Electromagnetic vertex}

The electromagnetic vertex $J_{\mu \nu}^{\rho}$ we use to calculate the deuteron form factors corresponds to the sum of the impulse approximation (IA) (figure 1) and of the contact interaction (C) (figure 2) which takes partially into account the two-body current:

$$
J_{\mu \nu}^{\rho}=J_{\mu \nu}^{\rho}(\mathrm{IA})+J_{\mu \nu}^{\rho}(\mathrm{C}) .
$$

These contributions are explained in what follows. 
The deuteron IA electromagnetic vertex is shown in figure 1. The dashed line is associated with a fictitious particle - the so called spurion. It reflects the fact that, although all the momenta are on the corresponding mass shells, the wave function is off energy shell, and therefore there is no any conservation law between all the components of the nucleon and the deuteron four-momenta. The spurion momentum just absorbs their nonzero difference and one has $k_{1}+k_{2}-p=\omega \tau$. To avoid misunderstanding, we emphasize that the spurion line does not imply the presence of an extra particle in the intermediate state in figure 1 which contains two nucleons only.

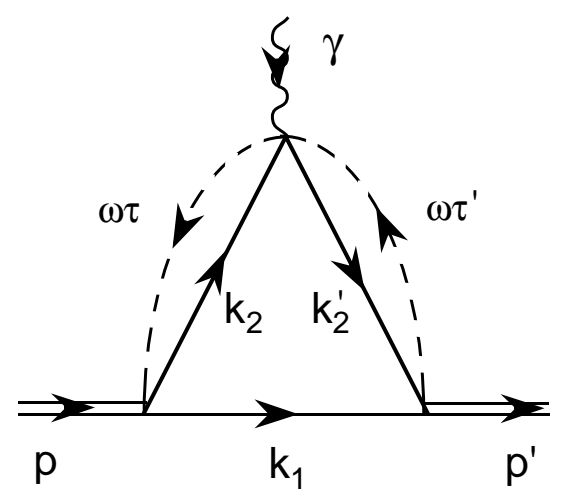

Figure 1: Electromagnetic vertex of the deuteron.

The electromagnetic amplitude in the impulse approximation is derived by applying the rules of the graph techniques [5] to figure 11. It has the form:

$$
\begin{aligned}
& \left\langle\lambda^{\prime}\left|J^{\rho}(\mathrm{IA})\right| \lambda\right\rangle=e_{\lambda^{\prime}}^{* \mu}\left(p^{\prime}\right) J_{\mu \nu}^{\rho}(\mathrm{IA}) e_{\lambda}^{\nu}(p) \\
& J_{\mu \nu}^{\rho}(\mathrm{IA})=\frac{m}{(2 \pi)^{3}} \int \operatorname{Tr}\left[\phi_{\mu}^{\prime}\left(\hat{k}_{2}^{\prime}+m\right) \Gamma^{\rho}\left(\hat{k}_{2}+m\right) \phi_{\nu}\left(\hat{k}_{1}-m\right)\right] \frac{d^{3} k_{1}}{(1-x)^{2} 2 \varepsilon_{k_{1}}},
\end{aligned}
$$

where $x=\omega \cdot k_{1} / \omega \cdot p$. The wave function $\phi_{\nu}=\phi_{\nu}\left(k_{1}, k_{2}, p, \omega \tau\right)$ given by eq.(1) corresponds to the deuteron initial state, while $\phi_{\mu}^{\prime}=\phi_{\mu}\left(k_{1}, k_{2}^{\prime}, p^{\prime}, \omega \tau^{\prime}\right)$ corresponds to its final state. $\Gamma_{\rho}$ is the electromagnetic vertex of the nucleon:

$$
\Gamma^{\rho}=F_{1} \gamma^{\rho}+\frac{i F_{2}}{2 m} \sigma^{\rho \alpha} q_{\alpha}
$$

$F_{1}$ and $F_{2}$ being the nucleon elecromagnetic form factors.

The expression for the trace in (1) is obtained as follows. To each nucleon line in figure 1 ] we associate the LFD nucleon propagator, with the $N N \gamma$ vertex we associate expression (5), and with $d-N N$ vertices the deuteron wave functions. We get in this way the following product of spin matrices:

$$
e_{\lambda^{\prime}}^{* \mu}\left(p^{\prime}\right)\left\{\left[\gamma_{0} \phi_{\mu}^{\prime} U_{c} \gamma_{0}\right]^{\dagger}\left(\hat{k}_{2}^{\prime}+m\right) \Gamma^{\rho}\left(\hat{k}_{2}+m\right) \phi_{\nu} U_{c}\right\}_{\beta \alpha} e_{\lambda}^{\nu}(p)\left(\hat{k}_{1}+m\right)_{\beta \alpha}
$$

Factor $\{\ldots\}_{\beta \alpha}$ in (6) corresponds to the upper line of the diagram and the factor $\left(\hat{k}_{1}+m\right)_{\beta \alpha}$ corresponds to the lower line. We attach the deuteron wave function to the upper one. We keep in (6) the matrix indices $\alpha$ and $\beta$ explicitly. Since both nucleon lines are passed in the 
same direction, the order of indices $\beta, \alpha$ is the same. This means that one of the factors in (6) (we take the second one) is a transposed matrix. The factor $\left[\gamma_{0} \phi_{\mu}^{\prime} U_{c} \gamma_{0}\right]^{\dagger}$ originates from the conjugated final deuteron wave function:

$$
\left(\bar{u}\left(k_{2}^{\prime}\right) \phi_{\mu}^{\prime} U_{c} \bar{u}\left(k_{1}\right)\right)^{\dagger}=u\left(k_{1}\right)\left[\gamma_{0} \phi_{\mu}^{\prime} U_{c} \gamma_{0}\right]^{\dagger} u\left(k_{2}^{\prime}\right)=-u\left(k_{1}\right) \bar{\phi}_{\mu}^{\prime} u\left(k_{2}^{\prime}\right),
$$

where $\bar{\phi}=\gamma_{0} \phi^{\dagger} \gamma_{0}$. With the wave function (1) we get:

$$
\left[\gamma_{0} \phi_{\mu}^{\prime} U_{c} \gamma_{0}\right]^{\dagger}=-U_{c} \phi_{\mu}^{\prime}
$$

and, hence, obtain the factor:

$$
-U_{c}\left(\hat{k}_{1}+m\right)^{t} U_{c}=\left(\hat{k}_{1}-m\right),
$$

that gives the trace in eq.(四). A more detailed derivation of (困) by means of the LFD graph technique can be found in [5]. Next section is devoted to the derivation of the contact contributions.

\section{The contact interaction}

A peculiarity of the LFD is the existence, in addition to the impulse approximation, of the so called instantaneous (or contact) interaction in the $N N B \gamma$ vertex, where $B$ is any of the mesons $\pi, \rho, \ldots$, building the NN potential. Its contribution to the electromagnetic vertex in $g^{2}$ order is shown in figure 2. The cross on a fermion line means that this line is not to be associated with a propagator, but with a factor proportional to $\hat{\omega}=\omega_{\mu} \gamma^{\mu}$. In the standard approach this is the well known instantaneous interaction vertex $\gamma^{\dagger}$. The nucleon electromagnetic vertex $\Gamma_{\rho}$ is determined, as usual, by the two nucleon electromagnetic form factors given in eq.(5).

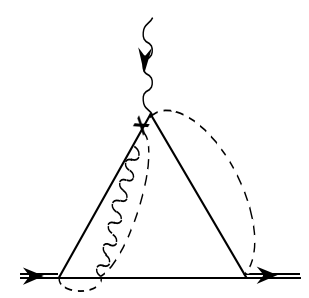

(a)

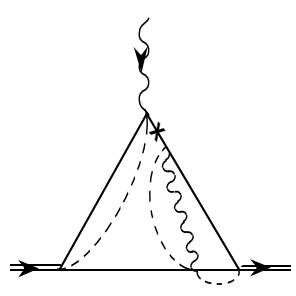

(c)

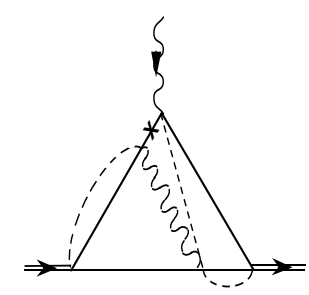

(b)

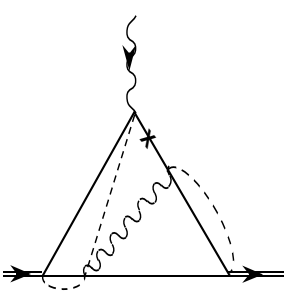

(d)

Figure 2: Contact term contributions to the electromagnetic interactions with deuteron.

In evaluating the contact amplitude, we have taken into account the sum over all six mesons contributing to the Bonn potential, with the parameters used in the Bonn model [6]. 
This amplitude incorporates partially the two-body currents. In principle more complicated diagrams, of order higher than $g^{2}$, may also contribute. Since our wave function takes into account corrections associated with the first degree of $g^{2}$, we keep the same order for the electromagnetic vertex. This means that we consider only the diagrams of figure 2 and in calculating these diagrams we omit component $f_{5}$ in the wave function, since its contribution multiplied by the contact interaction is of higher order than $g^{2}$. To calculate the effects beyond the $g^{2}$ order, one has also to take into account the higher order irreducible contributions to the OBE kernel. One can expect that these contributions are incorporated in this kernel on a phenomenological level by an appropriate fit of its parameters (coupling constants, meson masses and cutoff parameters). However, this is not the case for the electromagnetic vertex.

The contact amplitude is given by the sum of two terms which differ by the relative time order of the contact and the electromagnetic vertices:

$$
J_{\mu \nu}^{\rho}(\mathrm{C})=\left.J_{\mu \nu}^{\rho}(\mathrm{C})\right|_{l e f t}+\left.J_{\mu \nu}^{\rho}(\mathrm{C})\right|_{\text {right }} .
$$

The $\left.J_{\mu \nu}^{\rho}(\mathrm{C})\right|_{\text {left }}$ term corresponds to the sum of diagrams (a) and (b) in figure 2, which differ from each other by the relative time order of the meson vertices. Both of them have the contact vertex on the left hand side of the electromagnetic one. The opposite order is assumed in $\left.J_{\mu \nu}^{\rho}(\mathrm{C})\right|_{\text {right }}$ which corresponds to diagrams (c) and (d) in figure 2. In addition one has to take the sum over all mesons contributing to the interaction.

For a given meson, the amplitude corresponding to the sum of diagrams (a) and (b) in figure 2 - left contact term - has the form:

$$
\begin{array}{r}
\left.J_{\mu \nu}^{\rho}\right|_{l e f t}=m \int \operatorname{Tr}\left\{\left(-U_{c} \bar{\phi}_{\mu}^{\prime}\right)\left(\hat{k}_{2}^{\prime}+m\right) \Gamma^{\rho}\left(-\frac{\hat{\omega}}{2 \omega \cdot k_{2}^{\prime}}\right) V\left(\hat{k}_{2}+m\right) \phi_{\nu} U_{c}\right. \\
\left.\times\left[\left(\hat{k}_{1}^{\prime}+m\right) V\left(\hat{k}_{1}+m\right)\right]^{t}\right\} \frac{1}{\left(\mu^{2}+\vec{K}^{2}\right)} \frac{d^{3} k_{1}}{(2 \pi)^{3}(1-x) 2 \varepsilon_{k_{1}}} \frac{d^{3} k_{1}^{\prime}}{(2 \pi)^{3}\left(1-x^{\prime}\right) 2 \varepsilon_{k_{1}^{\prime}}}
\end{array}
$$

where $x=\omega \cdot k_{1} / \omega \cdot p, x^{\prime}=\omega \cdot k_{1}^{\prime} / \omega \cdot p$. The factor $\left(\mu^{2}+\vec{K}^{2}\right)$ is the denominator of the meson propagator, which is expressed in terms of the relative nucleon momenta. In these variables $\vec{K}^{2}$ is given by

$$
\vec{K}^{2}=\left(\vec{k}^{\prime}-\vec{k}\right)^{2}-\left(\vec{n} \cdot \vec{k}^{\prime}\right)(\vec{n} \cdot \vec{k}) \frac{\left(\varepsilon_{k^{\prime}}-\varepsilon_{k}\right)^{2}}{\varepsilon_{k^{\prime}} \varepsilon_{k}}+\left(\varepsilon_{k^{\prime}}^{2}+\varepsilon_{k}^{2}-\frac{1}{2} M^{2}\right)\left|\frac{\vec{n} \cdot \vec{k}^{\prime}}{\varepsilon_{k^{\prime}}}-\frac{\vec{n} \cdot \vec{k}}{\varepsilon_{k}}\right|
$$

In the time ordered graph technique, the analytical expressions for the diagrams (a) and (b) in figure 2 differ by the meson propagators. The modulus in (10) just take into account this change of the meson propagator for different time ordering of the vertices. Therefore expression (9) with (10) for $\vec{K}^{2}$ corresponds to the sum of graphs (a) and (b).

Expression (9) is obtained similarly to the case of the impulse approximation. The factor $V$ stands for the meson-nucleon vertex at the upper and lower lines. The factor $\left(-U_{c} \bar{\phi}_{\mu}^{\prime}\right)$ appears from relation (17). Taking again into account the fact that $U_{c} \hat{k}^{t}=-\hat{k} U_{c}$ we get:

$$
\begin{array}{r}
\left.J_{\mu \nu}^{\rho}\right|_{\text {left }}=\frac{m}{2^{7} \pi^{6}(\omega \cdot p)} \int \operatorname{Tr}\left\{\phi_{\mu}^{\prime}\left(\hat{k}_{2}^{\prime}+m\right) \Gamma^{\rho} \hat{\omega} V\left(\hat{k}_{2}+m\right) \phi_{\nu}\left(\hat{k}_{1}-m\right) V^{c}\left(\hat{k}_{1}^{\prime}-m\right)\right\} \\
\frac{1}{\left(\mu^{2}+\vec{K}^{2}\right)} \frac{1}{\left(1-x^{\prime}\right)} \frac{d^{3} k}{\varepsilon_{k}} \frac{d^{3} k^{\prime}}{\varepsilon_{k^{\prime}}}
\end{array}
$$


where $V^{c}=U_{c} V^{t} U_{c}$. We used the relation: $\omega \cdot k_{2}^{\prime}=\left(1-x^{\prime}\right) \omega \cdot p$. Instead of momenta $\vec{k}_{1}, \vec{k}_{2}$ we integrate in (11) over the relative nucleon momenta $\vec{k}, \vec{k}^{\prime}$, using the relation:

$$
\frac{d^{3} k_{1}}{2(1-x) \varepsilon_{k_{1}}}=\frac{d^{3} k}{\varepsilon_{k}}=\frac{d^{2} R_{\perp} d x}{2 x(1-x)}
$$

and similarly for $k_{1}^{\prime}, \vec{k}^{\prime}, \vec{R}_{\perp}^{\prime}$. These relative momenta are the arguments of the initial and final deuteron wave functions respectively.

The right contact term is obtained from eq.(11) by the replacement

$$
\frac{\Gamma^{\rho} \hat{\omega} V}{\left(1-x^{\prime}\right)} \rightarrow \frac{V \hat{\omega} \Gamma^{\rho}}{(1-x)}
$$

In case of scalar (pseudoscalar) exchanges one should put both for up and down vertices $V=V^{c}=g\left(V=V^{c}=i g \gamma_{5}\right)$. For the pseudoscalar exchange one can simplify the contact terms by excluding $\gamma_{5}$ :

$$
\begin{array}{r}
\left.J_{\mu \nu}^{\rho}\right|_{l e f t} ^{p s}=-\frac{m}{2^{7} \pi^{6}(\omega \cdot p)} \int \operatorname{Tr}\left\{\phi_{\mu}^{\prime}\left(\hat{k}_{2}^{\prime}+m\right) \Gamma^{\rho} \hat{\omega}\left(\hat{k}_{2}-m\right) \tilde{\phi}_{\nu}\left(\hat{k}_{1}+m\right)\left(\hat{k}_{1}^{\prime}-m\right)\right\} \\
\times \frac{g^{2}}{\left(\mu^{2}+\vec{K}^{2}\right)} \frac{1}{\left(1-x^{\prime}\right)} \frac{d^{3} k}{\varepsilon_{k}} \frac{d^{3} k^{\prime}}{\varepsilon_{k^{\prime}}}, \\
\left.J_{\mu \nu}^{\rho}\right|_{\text {right }} ^{p s}=-\frac{m}{2^{7} \pi^{6}(\omega \cdot p)} \int \operatorname{Tr}\left\{\tilde{\phi}_{\mu}^{\prime}\left(\hat{k}_{2}^{\prime}-m\right) \hat{\omega} \Gamma^{\rho}\left(\hat{k}_{2}+m\right) \phi_{\nu}\left(\hat{k}_{1}-m\right)\left(\hat{k}_{1}^{\prime}+m\right)\right\} \\
\times \frac{g^{2}}{\left(\mu^{2}+\vec{K}^{2}\right)} \frac{1}{(1-x)} \frac{d^{3} k}{\varepsilon_{k}} \frac{d^{3} k^{\prime}}{\varepsilon_{k^{\prime}}},
\end{array}
$$

where $\tilde{\phi}=\gamma_{5} \phi \gamma_{5}$. The function $\tilde{\phi}$ differs from $\phi$, eq.(看), by changing the sign of $\varphi_{2}$ (and also of $\varphi_{4}$ and $\varphi_{6}$ if they are not neglected).

Let us now find the vertices $V$ and $V^{c}$ for the vector exchange. It is convenient to take them from the expression of the corresponding kernel which has the form [5]:

$$
\begin{array}{r}
K=\int \bar{u}_{2}^{\prime}\left(g \gamma^{\alpha}-\frac{f}{2 m} \sigma^{\alpha^{\prime} \alpha} i\left(k-\omega \tau_{1}\right)_{\alpha^{\prime}}\right) \\
u_{2}\left[-g_{\alpha \beta}+\frac{\left(k-\omega \tau_{1}\right)_{\alpha}\left(k-\omega \tau_{1}\right)_{\beta}}{\mu^{2}}\right] \\
\times \bar{u}_{1}^{\prime}\left(g \gamma^{\beta}+\frac{f}{2 m} \sigma^{\beta^{\prime} \beta} i\left(k-\omega \tau_{1}\right)_{\beta^{\prime}}\right) u_{1} \\
\times \delta\left(\left(k_{1}-k_{1}^{\prime}+\omega \tau_{1}-\omega \tau\right)^{2}-\mu^{2}\right) \theta\left(\omega \cdot\left(k_{1}-k_{1}^{\prime}\right)\right) \frac{d \tau_{1}}{\tau_{1}-i \epsilon} \\
+\int \bar{u}_{2}^{\prime}\left(g \gamma^{\alpha}+\frac{f}{2 m} \sigma^{\alpha^{\prime} \alpha} i\left(k-\omega \tau_{1}\right)_{\alpha^{\prime}}\right) u_{2}\left[-g_{\alpha \beta}+\frac{\left(k-\omega \tau_{1}\right)_{\alpha}\left(k-\omega \tau_{1}\right)_{\beta}}{\mu^{2}}\right] \\
\times \bar{u}_{1}^{\prime}\left(g \gamma^{\beta}-\frac{f}{2 m} \sigma^{\beta^{\prime} \beta} i\left(k-\omega \tau_{1}\right)_{\beta^{\prime}}\right) u_{1} \\
\times \delta\left(\left(k_{1}^{\prime}-k_{1}+\omega \tau_{1}-\omega \tau^{\prime}\right)^{2}-\mu^{2}\right) \theta\left(\omega \cdot\left(k_{1}^{\prime}-k_{1}\right)\right) \frac{d \tau_{1}}{\tau_{1}-i \epsilon},
\end{array}
$$

$k$ is the meson momentum. The coupling constant $g$ stands for the vector vertex $\bar{u} \gamma^{\alpha} u$, whereas $f$ corresponds to the derivative coupling. The vector meson exchange generates its own vector 
contact interaction, corresponding to the cross on the vector meson line. This is an additional contact interaction in the vertex $N N \gamma V$. As indicated in [5], the contact term can be taken into account by the replacement, in the numerator, of the momentum $k$ by the difference $k-\omega \tau$, where $\omega \tau$ is the momentum of the spurion line connecting the ends of the vector meson line. This replacement has been done in (16). The factor $\omega \tau_{1}$ everywhere in $\left(k-\omega \tau_{1}\right)$ incorporates the contact terms both for the vector meson exchange and for the derivative coupling. We express $k$ in terms of the lower vertex momenta:

$$
\begin{aligned}
& k-\omega \tau_{1}=k_{1}-k_{1}^{\prime}-\omega \tau \quad \text { for } \quad \omega \cdot\left(k_{1}-k_{1}^{\prime}\right)>0 \\
& k-\omega \tau_{1}=k_{1}^{\prime}-k_{1}-\omega \tau^{\prime} \quad \text { for } \quad \omega \cdot\left(k_{1}^{\prime}-k_{1}\right)>0
\end{aligned}
$$

The first line of (17) corresponds to the first item in (16), the second line corresponds to the second one.

By this way, we find the vector contact term (both the left and the right one). It is convenient to represent it as the sum of two contributions:

$$
\left.J_{\mu \nu}^{\rho}\right|^{\text {vector }}=J_{\mu \nu}^{(1) \rho}+J_{\mu \nu}^{(2) \rho} .
$$

The first one, $J_{\mu \nu}^{(1) \rho}$, arises from the contraction of the $N N$-meson vertices with $-g_{\alpha \beta}$ in the meson propagator appearing in (16). For example, $J_{l e f t}$ is obtained by the following substitution in eq.(11) (and similarly for $J_{\text {right }}$ ):

$$
\begin{gathered}
V=g \gamma^{\alpha}-\frac{f}{2 m} \sigma^{\alpha^{\prime} \alpha} i\left(k_{1}-k_{1}^{\prime}-\omega \varphi\left(\tau, \tau^{\prime}\right)\right)_{\alpha^{\prime}}, \\
V^{c} \rightarrow g \gamma_{\alpha}+\frac{f}{2 m} \sigma_{\beta^{\prime} \alpha} i\left(k_{1}-k_{1}^{\prime}-\omega \varphi\left(\tau, \tau^{\prime}\right)\right)^{\beta^{\prime}} .
\end{gathered}
$$

The vertex $V^{c}$ is given by (20) with a minus sign; this sign is compensated by the minus from $-g_{\alpha \beta}$, what gives the substitution (20). In eqs.(19,20) we introduce the function, which takes into account the condition (17):

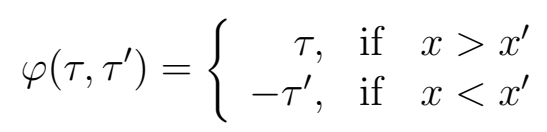

Since the vertex $V^{c}$ appears multiplied on the left by $\left(\hat{k}_{1}-m\right)$ and on the right by $\left(\hat{k}_{1}^{\prime}-m\right)$, it can be transformed into:

$$
V^{c} \rightarrow(g+f) \gamma_{\alpha}-\frac{f}{2 m}\left(k_{1}+k_{1}^{\prime}\right)_{\alpha}-i \frac{f}{2 m} \sigma_{\beta^{\prime} \alpha} \omega^{\beta^{\prime}} \varphi\left(\tau, \tau^{\prime}\right)
$$

The second item $J_{\mu \nu}^{(2) \rho}$ in $(18)$ arises from the contraction of the vertices with the factor $\left(k-\omega \tau_{1}\right)_{\alpha}\left(k-\omega \tau_{1}\right)_{\beta}$ in (16). It is given by (11) with the following vertices

$$
V \rightarrow \frac{g}{\mu}\left(\hat{k}_{1}-\hat{k}_{1}^{\prime}\right), \quad V^{c} \rightarrow \frac{g}{\mu} \hat{\omega} \varphi\left(\tau, \tau^{\prime}\right)
$$

The other terms, like the term proportional to $\hat{\omega}$, do not contribute to the upper vertex, since being multiplied by $\hat{\omega}$ from the contact term it gives zero. The difference $\hat{k}_{1}-\hat{k}_{1}^{\prime}$ in the lower vertex appears in the expression $\left(\hat{k}_{1}-m\right)\left(\hat{k}_{1}-\hat{k}_{1}^{\prime}\right)\left(\hat{k}_{1}^{\prime}-m\right)=0$ and, hence, does not contribute as well. The minus sign from $U_{c} \gamma_{\beta}^{t} U_{c}=-\gamma_{\beta}$ is incorporated in the function (21). 


\section{$5 \quad$ Calculating form factors}

The next step consists in extracting the deuteron form factors from the electromagnetic vertex $J_{\mu \nu}^{\rho}$. As already mentioned, in contrast to the wave function which is always off energy shell, the on-shell amplitudes should not depend on the light-front plane orientation. However, in practice, due to the incompatibility of the transformation properties of the approximate current and wave function, the nonphysical $\omega$ dependence survives in the on-energy shell deuteron electromagnetic vertex. Thanks to covariance, the general form of this dependence can be found explicitly [19]:

$$
\begin{aligned}
J_{\mu \nu}^{\rho} & =P^{\rho}\left[\mathcal{F}_{1} g_{\mu \nu}+\mathcal{F}_{2} \frac{q_{\mu} q_{\nu}}{2 M^{2}}\right]+\mathcal{G}_{1}\left(g_{\mu}^{\rho} q_{\nu}-g_{\nu}^{\rho} q_{\mu}\right) \\
& +B_{1} \frac{M^{2} \omega^{\rho} g_{\mu \nu}}{2 \omega \cdot p}+B_{2} \frac{\omega^{\rho} q_{\mu} q_{\nu}}{2 \omega \cdot p}+\cdots+B_{8} q^{\rho} \frac{q_{\mu} \omega_{\nu}+q_{\nu} \omega_{\mu}}{2 \omega \cdot p} .
\end{aligned}
$$

Here $q=p^{\prime}-p, P=p+p^{\prime}$, with $p$ and $p^{\prime}$ the initial and final deuteron momenta. As an example, we keep in (24) three $\omega$-dependent terms only, though the total number of them is eight. Since we assume $\omega \cdot q=0$ (what corresponds to $q_{+}=0$ in the standard approach), all the form factors in (24) depend on $Q^{2}=-q^{2}$ only.

The $\omega$-dependence of the wave function is not the only source of the nonphysical contributions $B_{1-8}$. With an $\vec{n}$-independent wave function (neglecting $f_{5}$ in (2)) we still get the $\omega$-dependent deuteron electromagnetic vertex. On the contrary, the physical form factors $\mathcal{F}_{1}, \mathcal{F}_{2}, \mathcal{G}_{1}$ do not depend on $\omega$ even with an $\omega$-dependent wave function. We emphasize that the physical $\omega$-dependent extra components in the wave function (2) and the nonphysical $\omega$-depending structures in (24) are present not because of the covariant formulation of the LFD. Their counterparts appear in the non-covariant light-front approach too but the covariant approach allows to indicate them explicitly.

The explicit formulas to extract the physical form factors $\mathcal{F}_{1}, \mathcal{F}_{2}, \mathcal{G}_{1}$ from the electromagnetic vertex and to separate them from nonphysical contributions $B_{1-8}$ were derived in refs. [19]. As an example, we give here the formula for $\mathcal{F}_{1}$ only:

$$
\mathcal{F}_{1}=J_{\mu \nu}^{\rho} \frac{\omega_{\rho}}{2 \omega \cdot p}\left[g^{\mu \nu}-\frac{q^{\mu} q^{\nu}}{q^{2}}-\frac{P^{\mu} \omega^{\nu}+P^{\nu} \omega^{\mu}}{2 \omega \cdot p}+P^{2} \frac{\omega^{\mu} \omega^{\nu}}{4(\omega \cdot p)^{2}}\right],
$$

The contraction with $\omega_{\rho}$ in (25) corresponds in the standard approach to the component $J_{\mu \nu}^{+}$. The expression for $\mathcal{F}_{2}$ is proportional to the same contraction. However the expression for $\mathcal{G}_{1}$ contains not only the contraction with $\omega_{\rho}$ but also others terms. Hence, the $J^{+}$component is not enough to find $\mathcal{G}_{1}$.

One can easily check, using $\omega \cdot q=0$, that one has for the nucleon electromagnetic vertex (5) $\hat{\omega} \omega_{\rho} \Gamma^{\rho}=\omega_{\rho} \Gamma^{\rho} \hat{\omega}=0$. Here $\hat{\omega}$ appears from the contact interaction, the contraction $\omega_{\rho} \Gamma^{\rho}$ is the result of eq.(25). Therefore the contact terms do not contribute to $\mathcal{F}_{1}, \mathcal{F}_{2}$, due to the contraction with $\omega_{\rho}$ in (25). They contribute only to $\mathcal{G}_{1}$. The form factors found this way are still not exact, but they are separated from the non-physical ones $B_{1-8}$. As shown in [19] and in [20], this separation is important, since the admixture of $B_{i}$ can considerably change the results.

After calculating traces and contracting tensors in eq.(25) for $\mathcal{F}_{1}$ (and similar expressions [5] for $\mathcal{F}_{2}$ and for $\mathcal{G}_{1}$ ) we obtain the integrand depending on the scalar products of the fourvectors $\omega, k_{1}, k_{2}, k_{1}^{\prime}, k_{2}^{\prime}, p, p^{\prime}, q$ with each other. In their turn, these scalar products, as well as 
the arguments of the wave functions, are expressed through the integration momenta and the momentum transfer. Their explicit kinematical formulas are given in [5]. The integrands also contain the scalar products with $\omega$ but after integration this dependence disappears.

By this way, substituting $J_{\mu \nu}^{\rho}$ in eq.(25) (and in similar expressions 19 for $\mathcal{F}_{2}, \mathcal{G}_{1}$ ), we find the analytical expressions of the form factors integrands. Then we carry out the numerical integration which, for the contact terms (two-loop), is 6-dimensional.

\section{Influence of the nucleon EM form factors}

Together with the deuteron wave function, an essential ingredient in these calculations are the nucleon electromagnetic form factors (NEMFF). The results presented in [5] were obtained with the dipole parametrization taken from Bilenkaya et al. [21] denoted hereafter by BKL. We analyse in what follows the influence of different NEMFF parametrizations in calculating the deuteron structure function within the same theoretical scheme. The parametrizations we consider, used in similar calculations found in the literature [12, 14, 26] 28], are those given by Galster et al. [22], Hohler et al. [23], a combination of Simon et al. for proton and Platchkov et al. for neutron (SP) [24] and the more recent publication of Mergell et al. (MMD) [25]. The comparison [29] of the proton $\left(G_{E}^{p}, G_{M}^{p}\right)$ and neutron $\left(G_{E}^{n}, G_{M}^{n}\right)$ charge and magnetic form factors with the existing experimental data [30] is shown in figure 3. The values of the neutron charge form factor have been squared to account for the results reported in [30]. A first sight to this figure shows that some of these form factors have been apparently determined in the low momentum region and can not be used above $1-2(\mathrm{GeV} / \mathrm{c})^{2}$. The more adequated parametrization covering the whole momentum region seems to be MMD [25], which will be adopted in our calculations. It provides an acceptable description for all the form factors although some variations can not be excluded due to the inaccuracy of the existing measurements, especially for the neutron form factor (see below). Any comparison between the theoretical and experimental results has to be understood in the context of this uncertainty that we would like to estimate.

The influence of different NEMFF parametrizations on the deuteron structure function $A\left(Q^{2}\right)$ is shown in figure 4 . The results in figure $4(\mathrm{a})$ are fully non relativistic whereas those in $1(\mathrm{~b})$ correspond to LFD impulse approximation. The choice of the NEMFF has small influence at low momentum transfer. For instance at $Q^{2}=0.5(\mathrm{GeV} / \mathrm{c})^{2}$, the deviation in the nonrelativistic structure function $A$ is $\approx 15 \%$. This deviation increases with $Q^{2}$ and reaches already a factor two at $Q^{2}=2(\mathrm{GeV} / \mathrm{c})^{2}$ between BKL and SP parametrizations. The difference becomes dramatic for the last measured values $Q^{2}=6(\mathrm{GeV} / \mathrm{c})^{2}$ where there is one order of magnitude in the structure functions $A\left(Q^{2}\right)$ calculated using BKL or Hohler parametrizations. It is worth noticing however that the Galster and MMD sets lead to close results in all the momentum range. Their difference in the LFD-IA relativistic calculation of $A$ remains at the level of $10 \%$ for $Q^{2} \leq 10(\mathrm{GeV} / \mathrm{c})^{2}$. Similar deviations have been found in the structure function $B$.

As one can see from figure 3, the neutron electric form factor $G_{E}^{n}$ contains the greatest uncertainty. To estimate the influence of this uncertainty, we have considered for $Q^{2}=2-4$ $(\mathrm{GeV} / \mathrm{c})^{2}$ the maximal $G_{E}^{n^{2}}$ values compatible with the error bars, which are a priori not excluded by the experimental data although they considerably differ from all fits. The values of $A\left(Q^{2}\right)$ obtained with such a modification exceed by $20-30 \%$ those obtained with the original MMD parametrization. From these considerations it follows that $i$ ) the comparison between 


\section{Nucleons form factors, param SPA}
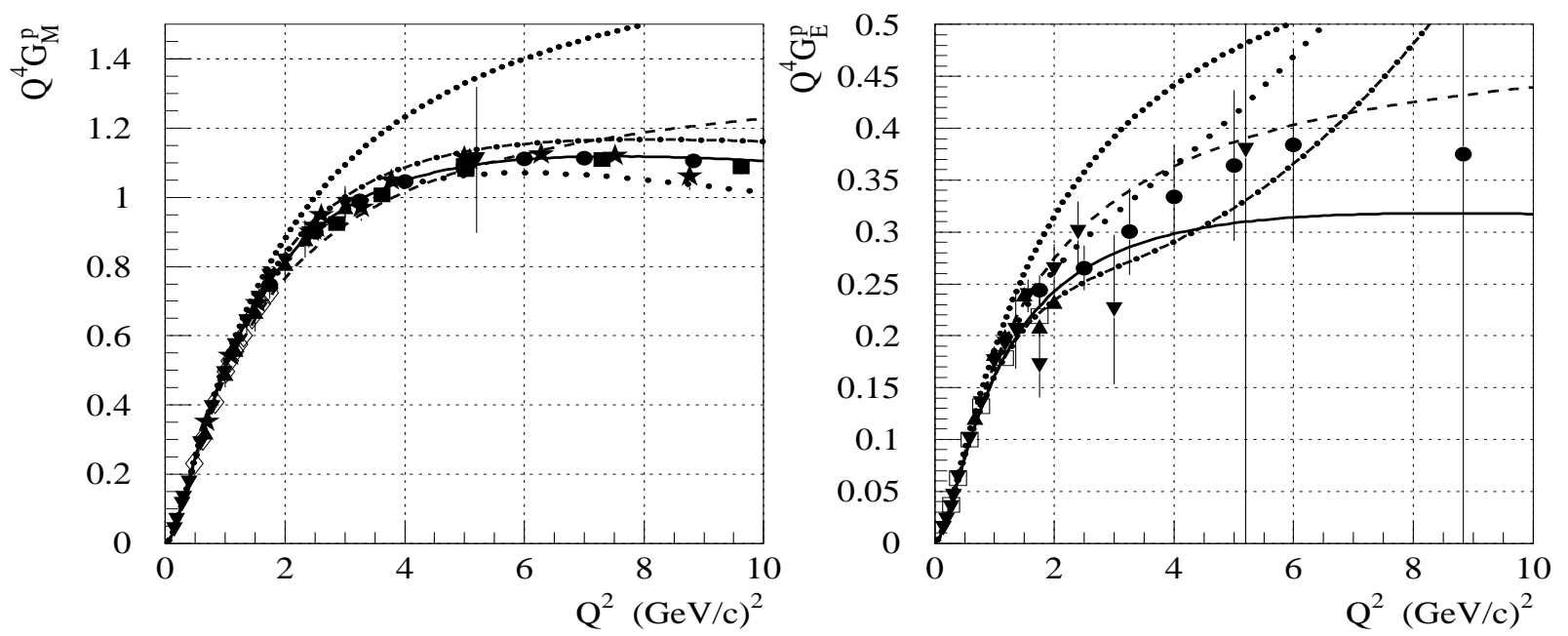

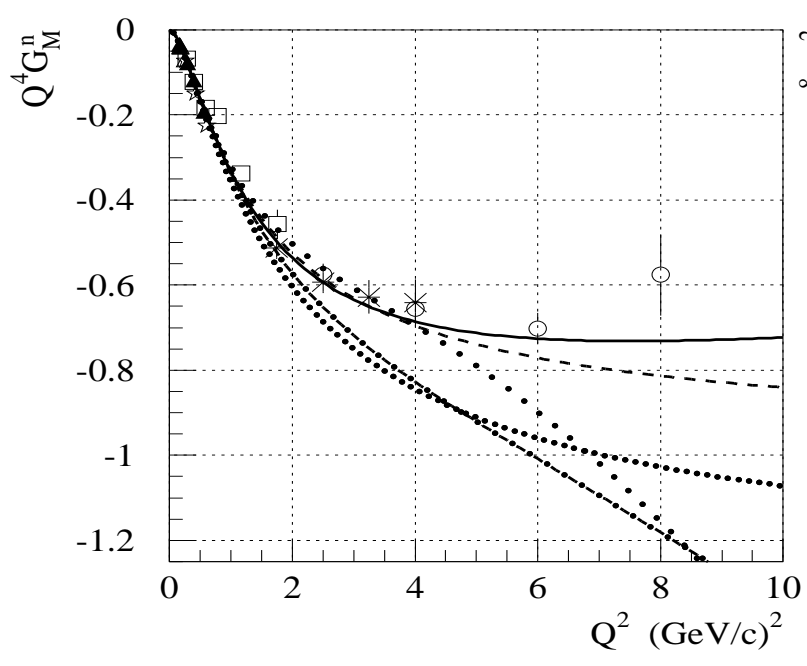

$\star$ Coward (1968)

- Bartel (1973)

$\square$ Hanson (1973)

จ Hohler (1976)

- Rock (1982)

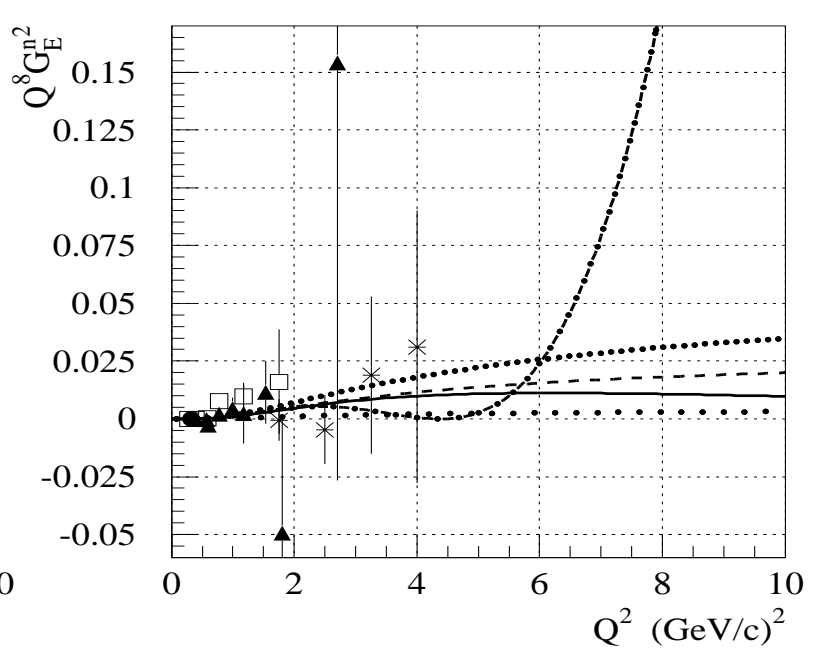

$\diamond$ Bosted (1990)

$\triangle$ Markowitz (1993)

* Lung (1993)

- Sill (1993)

¡ Nikhef 93

Figure 3: Comparison between proton $\left(G_{E, M}^{p}\right)$ and neutron $\left(G_{E, M}^{n}\right)$ electromagnetic form factors parametrizations and experimental data taken from [30]. The continuous line corresponds to MMD [25], dot-dashed (-.-.-) to Höhler et al. [23], dashed (- - ) to Galster et al. [22], short-dotted (...) to BKL [21] and long-dotted (. . .) to SP [24] 


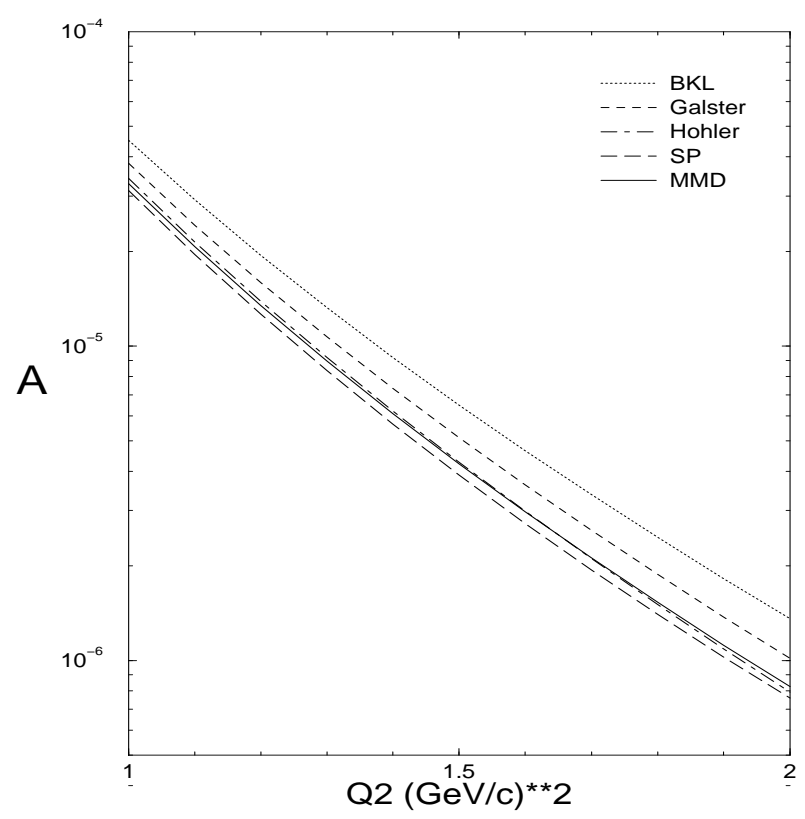

(a)

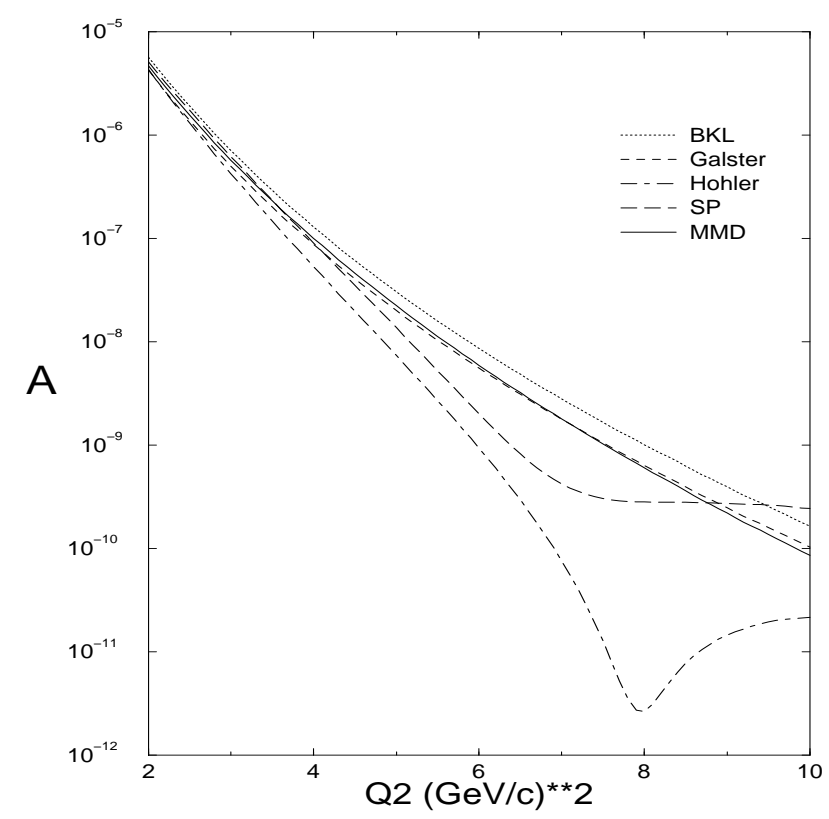

(b)

Figure 4: Influence of the nucleon electromagnetic form factors in the deuteron structure function $A\left(Q^{2}\right)$ in the non relativistic impulse approximation (a) and in the LFD impulse approximation (b)

different calculations is only meaningful when the same parametrization of NEMFF is used and ii) the comparison between a theoretical prediction and the measured deuteron form factors is limited by the considerable uncertainties implied by the poor knowledge of the NEMFF for $Q^{2}>1(\mathrm{GeV} / \mathrm{c})^{2}$.

\section{$7 \quad$ Results and discussion}

We present in this section the comparison of the deuteron structure functions and tensor polarization observable with the last measured values. In terms of the deuteron form factors these observables read

$$
\begin{aligned}
A\left(q^{2}\right) & =F_{C}^{2}\left(q^{2}\right)+\frac{8}{9} \eta^{2} F_{Q}^{2}\left(q^{2}\right)+\frac{2}{3} \eta F_{M}^{2}\left(q^{2}\right) \\
B\left(q^{2}\right) & =\frac{4}{3} \eta(1+\eta) F_{M}^{2}\left(q^{2}\right) \\
t_{20}\left(A\left(q^{2}\right)+\tan ^{2} \frac{1}{2} \theta B\left(q^{2}\right)\right) & =-\frac{1}{\sqrt{2}}\left[\frac{8}{3} \eta F_{C} F_{Q}+\frac{8}{9} \eta^{2} F_{Q}^{2}+\frac{1}{3} \eta\left(1+2(1+\eta) \tan ^{2} \frac{1}{2} \theta\right) F_{M}^{2}\right]
\end{aligned}
$$

where

$$
F_{C}=-\mathcal{F}_{1}-\frac{2 \eta}{3}\left[\mathcal{F}_{1}+\mathcal{G}_{1}-\mathcal{F}_{2}(1+\eta)\right]
$$




$$
\begin{aligned}
F_{M} & =\mathcal{G}_{1}, \\
F_{Q} & =-\mathcal{F}_{1}-\mathcal{G}_{1}+\mathcal{F}_{2}(1+\eta),
\end{aligned}
$$

and $\eta=Q^{2} / 4 M^{2}$. In comparing our calculations with the data we should first emphasize that, though the theoretical framework we use - the light-front dynamics - is fully selfconsistent, the validity of our perturbative method to evaluate the wave function is restricted to relative nucleon momenta $k$ smaller than the nucleon mass $m$. Since in a form factor calculation the momentum transfer is distributed between two deuteron vertices, the initial and final ones, this value of $k$ corresponds approximately to the momentum transfer $Q^{2} \leq(2 m)^{2} \approx 3.5(\mathrm{GeV} / \mathrm{c})^{2}$. In absence of subtle cancellations increasing the uncertainty in the theoretical predictions, we can expect a reasonable description of the experimental data in this momentum region.
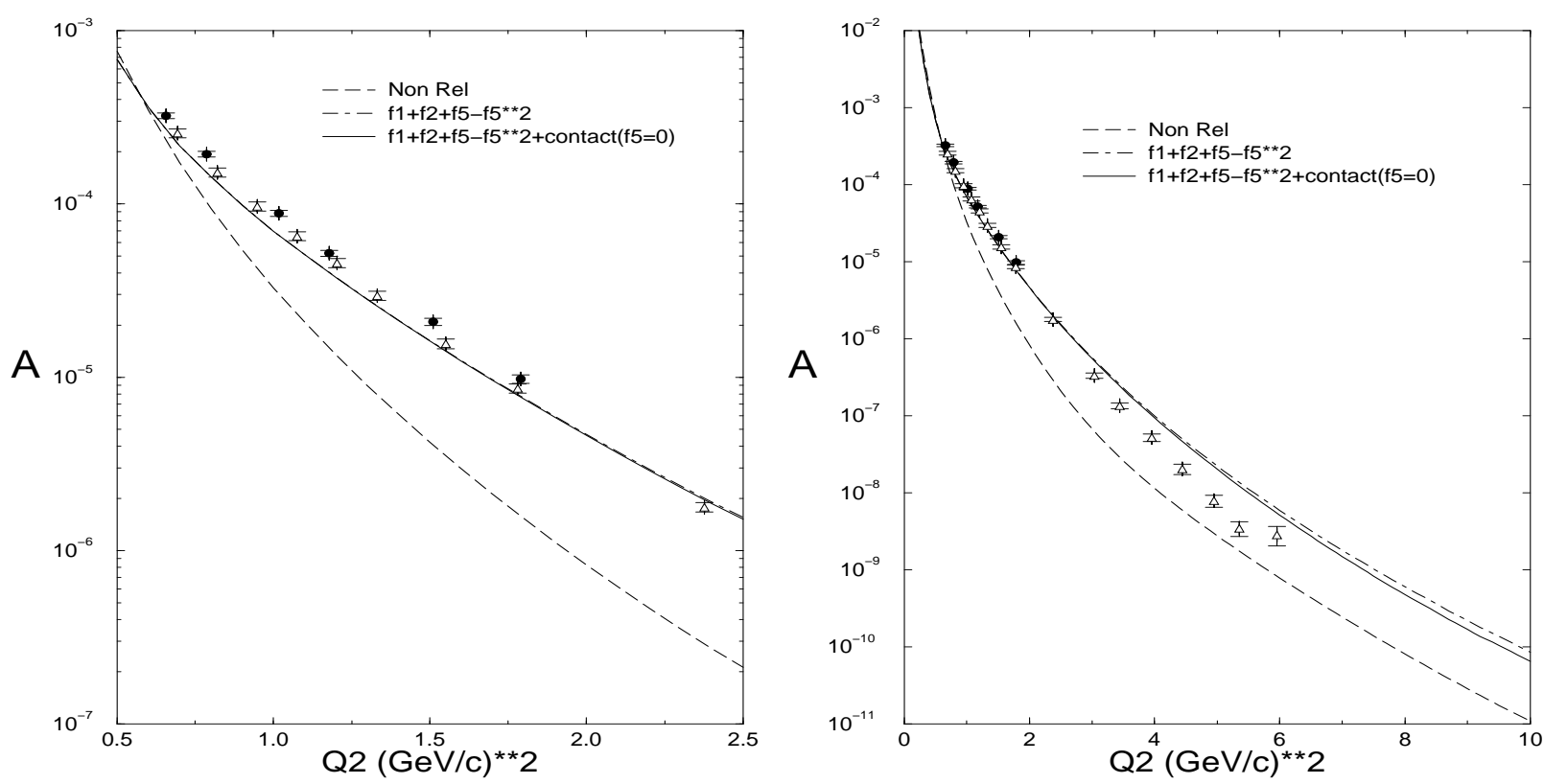

Figure 5: The structure function $A\left(Q^{2}\right)$ of the deuteron with MMD nucleon form factors. The curves are explained in the text.

In figure 5 the structure function $A\left(Q^{2}\right)$ is shown together with the experimental points recently obtained at TJNAF (Hall C in filled circles [1] and Hall A in opaque triangles up [2]). The dashed curve corresponds to the non-relativistic impulse approximation with the S- and Dwaves of the Bonn-QA wave function [6]. The dot-dashed line is also calculated in the impulse approximation but using the light-front formalism with relativistic deuteron components $f_{1}$ and $f_{2}$ and the $f_{5}$ component in first degree only. The solid line incorporates, in addition, the contact terms which turn to have small influence in this observable. We can see a good agreement until $Q^{2}=2.5(\mathrm{GeV} / \mathrm{c})^{2}$, a momentum region where the departure from a non relativistic description reaches one order of magnitude. It is worth noticing that this agreement is found with the LFD wave function alone, i.e. without explicitly including any MEC diagrams. However, as it has been already mentioned, the extra component $f_{5}$ accounts for the so called pair terms in the deuteron electrodisintegration amplitude [18]. We remark that a systematic deviation seems to manifest above $3(\mathrm{GeV} / \mathrm{c})^{2}$. This excess is smoothly increasing with increase of $Q^{2}$. However, this is the region where the perturbative calculation is hardly reliable, since the deuteron wave 
function appears in $A\left(Q^{2}\right)$ in the fourth degree and a $50 \%$ correction may change the value by a factor 5. Besides, as it has been shown above, at $Q^{2}>2(\mathrm{GeV} / \mathrm{c})^{2}$ the uncertainty coming from the NEMFF is also high.

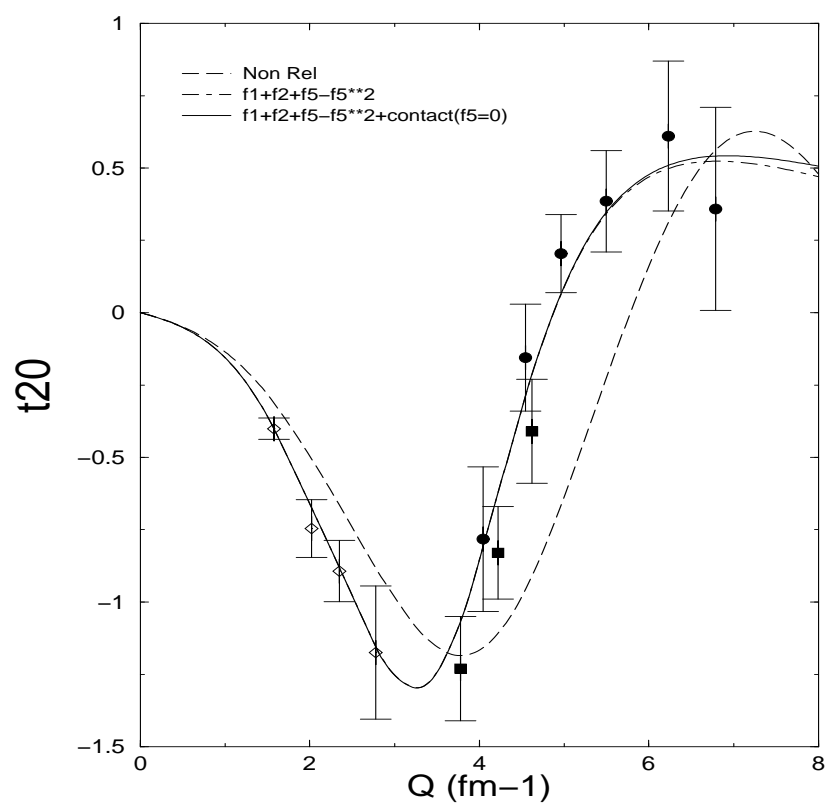

Figure 6: The deuteron tensor polarization $t_{20}$ at $\theta=70^{\circ}$. The designations of the curves are the same as in figs. 5 .

The same agreement is seen in the deuteron polarization observable $t_{20}$ displayed in figure 6 using the same drawing conventions. Again a sizeable effect of the relativistic corrections is properly taken into account in our calculations although the actual error bars are quite comfortable.

The calculated structure function $B\left(Q^{2}\right)$ is shown in figure [7, using the same drawing conventions, together with the experimental data from 31. Comparing the $B\left(Q^{2}\right)$ results with those of $A\left(Q^{2}\right)$ and $t_{20}$, one can see a considerable deviation of our $B$ calculation from the data, especially in the region of the minimum, as well as highest sensitivity to the different approximations. This minimum corresponds to the zero of the deuteron magnetic form factor $F_{M}$, and a small shift on this value drastically changes the value of $B\left(Q^{2}\right)$ in its neighborhood. It is important to emphasize that the zero of $F_{M}$ which exists in a nonrelativistic calculation, disappears for the relativistic $f_{1}, f_{2}$ and appears again when $f_{5}$ is taken into account [5]. In a relativistic framework this minimum is thus a consequence of a delicate cancellation between the $f_{5}$ contribution with the contributions of $f_{1}$ and $f_{2}$. None of these $f_{1}, f_{2}, f_{5}$ components has been calculated with enough accuracy in our perturbative approach. Therefore we cannot pretend to a detailed description of $B\left(Q^{2}\right)$ in this region.

The situation is different for $A\left(Q^{2}\right)$ and $t_{20}$. Figure 8 shows the relative contributions of the three deuteron form factors to these observables according to equations (26) and (28). One can see that their values are dominated by the charge and quadrupole form factors $F_{C}$ and $F_{Q}$, whereas the contribution of $F_{M}$, containing the highest uncertainty, is suppressed.

Taking into account the same order of accuracy, i.e., $g^{2}$, which was kept in the calculation of the wave function, we get a good description of the data on $t_{20}$ as well as of $A\left(Q^{2}\right)$ in the 


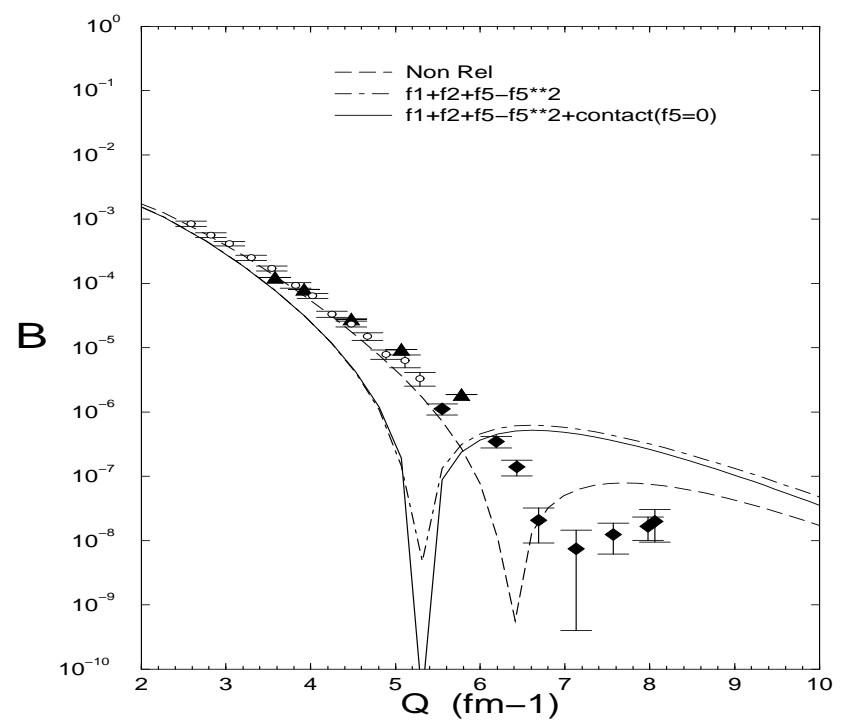

Figure 7: Same as figure 5 but for $B\left(Q^{2}\right)$.

same region of the momentum transfer, up to $2(\mathrm{GeV} / \mathrm{c})^{2}$. The relativistic effects in $f_{1}, f_{2}$ and the contribution of $f_{5}$ are important to achieve this good description. The contribution of the contact term to $A\left(Q^{2}\right)$ and $t_{20}$ is small, since, as noted above, it contributes to $F_{M}$ only, whereas for $B$ it is small in the momentum transfer region shown in figure 7 but increases at higher momenta (e.g. by a factor 2 at $\left.Q^{2}=6(\mathrm{GeV} / \mathrm{c})^{2}\right)$. These results show that the relativistic effects in the deuteron wave function, including the extra component $f_{5}$, and in the deuteron electromagnetic vertex make considerable influence on the deuteron form factors. Note that the effect of incorporating $f_{5}$ on $t_{20}$ is qualitatively similar to that obtained when adding the contribution of the pair current in non-relativistic calculations [27].

We should finally emphasize that the results presented in this section have been obtained without fitting any new set of parameters, neither in the nucleon-meson form factors nor in the $\mathrm{NN}$ interaction kernel, which was taken as it is given in ref. [6].

The agreement of our calculations with the experimental data, as well as with some other calculations carried out in the framework of meson-nucleon dynamics (see e.g. [7]-27]), shows that the deuteron structure at small distances is understood rather well within this theoretical framework. It is a remarkable fact that probing distances of the order of $0.1 \mathrm{fm}$, at which quarks effects should manifest themselves in their full glory, could be accounted by the relativistic nucleon-meson dynamics and the phenomenological nucleon form factors.

The parameters of the effective nucleon-meson Lagrangian, fixed by fitting the $N N$ experimental data, should be in principle derived from QCD, what is a separate problem. This allows us to make the following general conclusion: at least in what concerns the deuteron, the relativistic nuclear dynamics can be developed independently of its derivation from QCD.

Our calculations can be improved in many respects. An exact solution of the equation for the deuteron is necessary and is in progress. Besides the non-perturbative calculation, it implies the determination of the $N N$ kernel parameters in the framework of the light-front equations. This requires also a careful treatment of higher order contributions to the kernel. Finally, the calculation of electromagnetic observables should include higher Fock states $(N N \pi$, etc.) and the meson exchange currents which are not included in the wave function components, like 


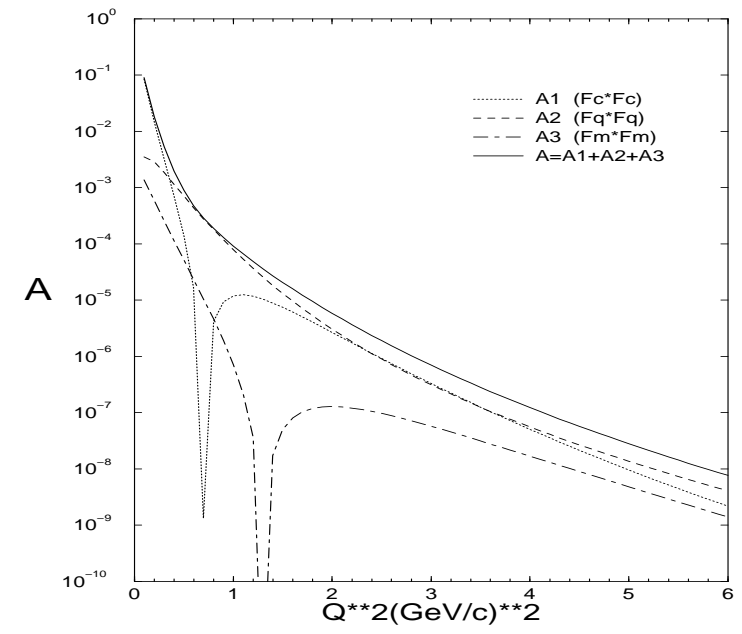

(a) $A\left(Q^{2}\right)$

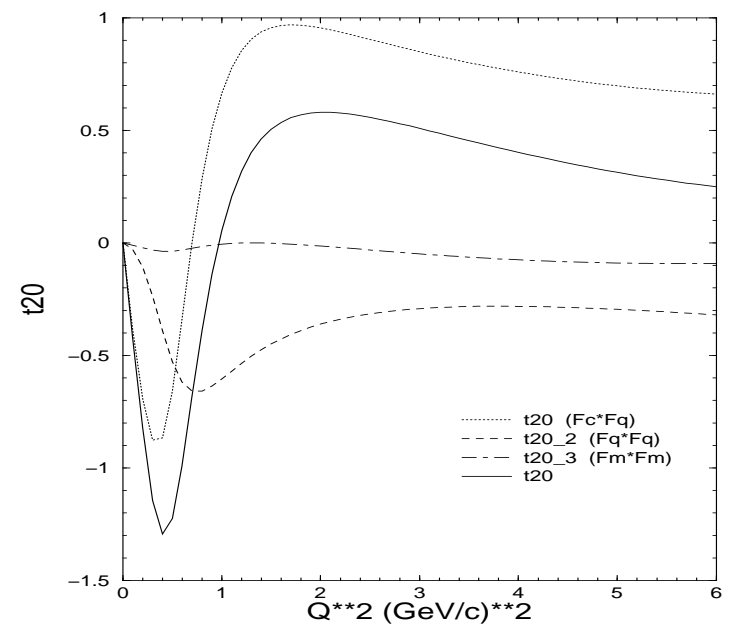

(b) $t_{20}$

Figure 8: The contributions of the different deuteron form factors $\left(F_{C}, F_{Q}, F_{M}\right)$ to the structure function $A\left(Q^{2}\right)$ (a) and $t_{20}(\mathrm{~b})$.

those corresponding to the interaction of the photon with the intermediate mesons $(\rho \pi \gamma, \ldots)$.

The improvement of the experimental data on the nucleon EM form factors is also an urgent task.

\section{Acknowledgements}

The authors are sincerely grateful to J.S. Real for providing the experimental compilation of the nucleon electromagnetic form factors, to B. Desplanques for stimulating critics and valuable remarks and to M. Mangin-Brinet for helpful discusions.

\section{References}

[1] D. Abbott et al, Jefferson Lab t20 Collaboration, Phys. Rev. Lett, 82 (1999)

[2] L.C. Alexa et al., Jefferson Lab Hall A Collaboration, Phys. Rev. Lett., 82 (1999)

[3] C. Furget et al., Acta Physica Polonica B 29 (1998) 3301

[4] B. Beise, Proceedings of XVI European Few-Body Conference, Autrans (France) 1998, to appear in Few-Body Systems (1999)

[5] J. Carbonell, B. Desplanques, V.A. Karmanov and J.-F. Mathiot, Phys. Reports, 300 (1998) 215.

[6] R. Machleidt, K. Holinde and Ch. Elster, Phys. Reports, 149 (1987) 1. 
[7] I.L. Grach and L.A. Kondratyuk, Yad. Phys. 39 (1984) 316 [Sov. J. Nucl. Phys. 39 (1984) 198]

[8] P.L. Chung, F. Coester, B.D. Keister and W.N. Polyzou, Phys. Rev. C37 (1988) 2000

[9] L.L. Frankfurt, I.L. Grach, L.A. Kondratyuk and M.I. Strikman, Phys. Rev. Lett. 62 (1989) 387

[10] L.L. Frankfurt, T. Frederico and M. Strikman, Phys. Rev. C48 (1993) 2182.

[11] M.J. Zuilhof and J.A. Tjon, Phys. Lett. 84B (1979) 3; Phys. Rev. C22 (1980) 2369; C24 (1981) 736; C26 (1982) 277.

[12] E. Hummel and J.A. Tjon, Phys. Rev. Lett., 63 (1989) 1788; Phys. Rev. C42 (1990) 423

[13] G. Arnold, C.E. Carlson and F. Gross, Phys. Rev. C21 (1980) 1426.

[14] J.W. Van Orden, N. Devine, F. Gross, Phys. Rev. Lett., 75 (1995) 4369

[15] F. Gross, Phys. Rev. 186 (1969) 1448; Phys. Rev. D10 (1974) 223; Phys. Rev. C26 (1982) 2203

[16] V.A. Karmanov, Nucl. Phys. A362 (1981) 331.

[17] J. Carbonell and V.A. Karmanov, Nucl. Phys. A581 (1995) 625.

[18] B. Desplanques, V.A. Karmanov and J.-F. Mathiot, Nucl. Phys. A589 (1995) 697.

[19] V.A. Karmanov and A.V. Smirnov, Nucl. Phys. A546 (1992) 691; A575 (1994) 520.

[20] V.A. Karmanov, Nucl. Phys. A608 (1996) 316.

[21] S.I. Bilenkaya, Yu.M. Kazarinov and L.I. Lapidus, ZhETF, 61 (1971) 2225 [Sov. Phys. JETP 34 (1972) 1192].

[22] S. Galster et al., Nucl. Phys. B32 (1971) 221

[23] G. Höhler et al., Nucl. Phys. B114 (1976) 505

[24] G.G. Simon et al., Nucl. Phys. A333 (1980) 381

S. Platchkov et al., Nucl. Phys. A510 (1990) 740

[25] P. Mergell, U-G. Meissner, D. Dreschell, Nucl. Phys. A596 (1996) 367

[26] R.B. Wiringa, V.G.J. Stoks, R. Schiavilla, Phys. Rev. C51 (1995) 38

[27] R. Schiavilla and D.O. Riska, Phys. Rev. C43 (1991) 437.

[28] A. Amghar, N. Aissat, B. Desplanques, Eur. Phys. J. A1 (1998) 85

[29] J.S. Real, private communication 
[30] D.H. Coward et al., Phys. Rev. Lett. 20 (1968) 292; W. Bartel et al., Nucl. Phys. B58 (1973) 429; K.M. Hanson et al., Phys. Rev. D8 (1973) 753; G. Höhler et al., Nucl. Phys. B114 (1976) 505; S. Rock et al., Phys. Rev. Lett. 49 (1982) 1138; P. Markowitz et al., Phys. Rev. C48 (1993) R5; A. Lung et al., Phys. Rev. Lett. 70 (1993) 718; A. F. Sill et al., Phys. Rev. D48 (1993) 29; M. Meyerhoff et al., Phys. Lett. B327 (1994) 201; E.E.W. Bruins et al., Phys. Rev. Lett. 75 (1995) 21

[31] R. Cramer et al, Z. Phys C29 (1985) 513

S. Auffret et al., Phys. Rev. Lett. 54 (1985) 649

P.E. Bosted et al., Phys. Rev. C42 (1990) 38 